\title{
Design and implementation of photo printing system using WeChat and electronic payment
}

\author{
Zhang Zhixin \\ Beijing University of Posts and Telecommunications \\ Beijing, China \\ uuytap@163.com
}

\author{
You Xiangdong \\ Beijing University of Posts and Telecommunications \\ Beijing, China \\ youxiangdong@bupt.edu.cn
}

\begin{abstract}
A real-time photo printing system is an innovative "Internet Plus" project, which is different from the traditional photo film industry. Users only need to speed a short period of time to complete the photo printing. At the same time, with the help of electronic payment, such as WeChat payment, Beijing traffic IC card and so on, the operation process becomes simple and easy. This Paper firstly introduces the background of the project, and then demonstrates the implementation of the whole system, shows the technical features and innovative points, next lists the results, and finally expounds the project's promote value and prospect of promotion as a conclusion.
\end{abstract}

Keywords—real-time photo printing; WeChat; Internet Plus;

\section{INTRODUCTION}

With the popularity of $4 \mathrm{G}$, the loss of the mobile phone flow rates and the popularity of Wi-Fi hotspots, mobile Internet technology is developing rapidly, an increasing number of "Internet Plus" project appears in our daily life. Meanwhile, with the abundance of electronic payment channels, as well as the increase of payment safety, this kind of new payment method is gradually replacing the traditional one, and will become the mainstream in the future. Among those "Internet Plus" project, the real-time photo printing system seize the user needs.

This project is aiming at a kind of "real-time photo printing client". With the help of WeChat Official Accounts, electronic payment, we implemented a set of printing system, which is easy to use. Users can upload photos in their own cellphones quickly by WeChat Official Accounts to web server. After cutting, beautification, they can use client to download the photo, and print it. Electronic payment will help them complete the process quickly. Users can print out 6 or 8 inches of photos even if 1 or 2 inches' ID photos. This project is suitable for using in schools, resorts, and office buildings.

This paper firstly introduces the background, then introduces the technical framework and the data structure, next analysis each technical points of the project, finally introduces the value of the project and the prospect of project's promotion.

\section{BACKGROUND}

\section{A. Analysis of photo printing market}

At present, with the improvement of mobile phone performance, more and more people prefer to share digital photographs rather than normal ones, mainly because film photos could be extremely expensive and will take several days before we can get them. However, some kind of photos, such as ID photos, are now in great demand and everyone will use them.

\section{B. Status of WeChat}

WeChat is an instant messaging app. It occupies a large market, and it is currently the most popular app in China. By the end of 2015, WeChat users has reached 549 million, covers more than $90 \%$ of the smartphone users [1]. Meanwhile, WeChat Official Accounts, has also became the strongest propagation channel, as well as the most influential media platform. This project is based on the WeChat Official Accounts, which help us realize the file uploading, photo cutting, payment, and some other functions.

\section{Status of Electronic Payment}

At present, headed by WeChat payment and Alipay, more and more electronic payment platform begin to affect our life. Especially in recent one or two years, with the rapid development of WeChat payment, most of the WeChat users has been bounded their bank cards to WeChat wallet, so that they can easily buy things, give money to their friends and so on. At the same time, in Beijing, traffic card is also a kind of mainstream payment tool and almost everyone has one. The system supports both the above two kinds of payment, thus greatly improved the efficiency.

\section{PRinciPle AND Method of Design}

\section{A. Framework}

The whole system, from a functional perspective, is divided into 5 different parts: photo downloading, photo beautification, electronic payment, statistics, and automation management. Cooperated with the server and the WeChat Official Accounts, the system, on the basis of realizing the function of the photo printing, can guarantee the program run stably for a long time.

The system runs on Windows 7, using the Python language and cooperate with the relevant third-party libraries to complete function. Using the Http as network communication protocols, Json as the data transmission tool. The whole system framework is shown in Fig. 1. 


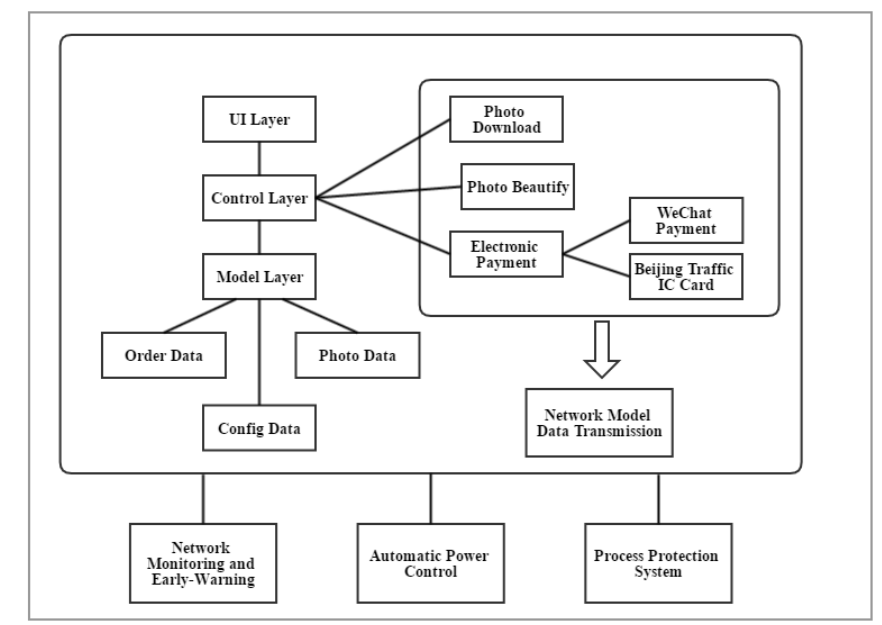

Fig. 1. Printint system framework

In this system, a complete process includes photo downloading, number choosing, photo beautifying (optional), paying, and last printing, Fig. 2 show the whole process of the system.

\section{B. Module Details}

This system adopts MVC framework, divided into multiple modules. Modules are combine with each other using a loosely coupled way. The details of the module are described below:

- UI layer is developed using PyQt. Qt is the graphical user interface framework developed in $\mathrm{C}++$, it uses an object-oriented design patterns, which has strong and perfect performance [2]. PyQt is a kind of Qt framework rewrite in Python. The entire UI layer using the signals and slots to handle all types of click and respond events, as well as screen switch and UI control, such as image, text, background, buttons and so on. The entire interface is running smoothly, sensitive and fast.

- Model layer using object-oriented principles, it's mainly purpose is managing transaction data, photo data, user data and system configuration data. It is connected with the control layer. The model layer is loosely coupled designed so that the control layer can be easily carried out on the data CRUD. Model layer is the base of whole system.

- Control layer is mainly responsible for the completion of specific logic functions and connect the data layer and the UI layer. Among them, the image processing section used Python PIL library, users can zoom in on the image, as well as crop, rotate, format conversion, filter and so on. In order to ensure the smooth operation feeling of the UI layer, namely no blocking occurs, the control layer maintains a queue using asynchronous thread to handle time-consuming operations such as network connections and database loading, thus improving the user experience.

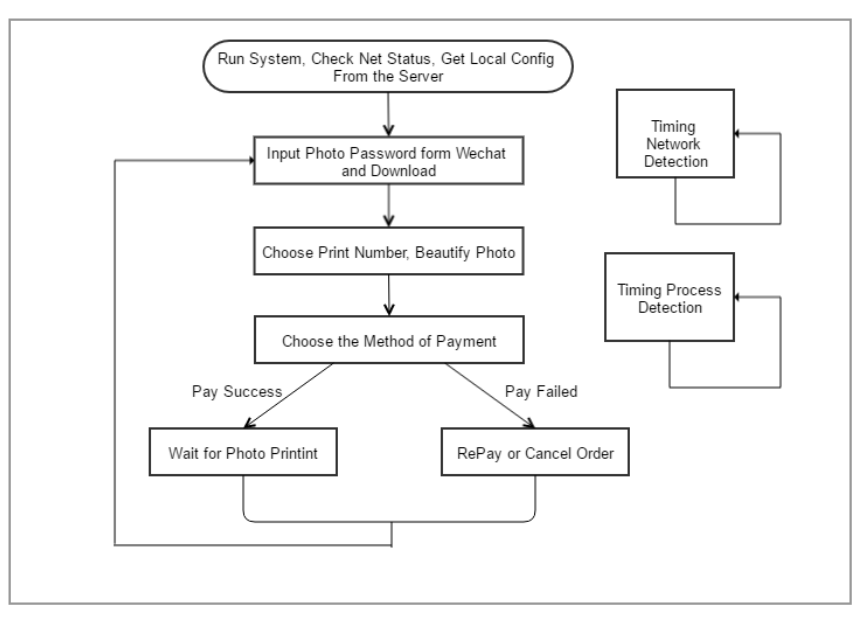

Fig. 2. The process of system

- Network module is responsible for connecting client and server, using python requests library. Clients can download photo, complete payment, confirm payment status and upload transaction order effectively. Taking into account that the real network conditions may be not that good. The module should endure fluctuation, timeout, terminals, and any other exceptions, making the whole system more robust.

On this basis, printing client also use a number of systemlevel tools, software and scripts to ensure the stability of the system.

\section{TECHINICAL FEATURES}

\section{A. Dynamic Parameter Configuration}

For clients in different regions, we may need to set different price and discount policies to adapt to different users. All configuration parameters in this system are managed by cloud server. Every time the client run, the program will firstly access the cloud server to get the latest configuration parameters, including the photo price, discount policy, root password, and so on. Administrators can easily change these parameters via website, thus making the system management more convenient.

\section{B. Process Protection System, Heartbeat}

For actual use printing system, the degree of redundancy mechanism is essential, in the case of extreme environments (such as severe fluctuations in the network, process dead or some undiscovered vulnerabilities). Without good fault tolerant mechanisms may lead to the collapse of the entire system. Therefore, in order to ensure system can run stable, we use a kind of process protection strategy called "watchdog" [3].

The "watchdog" will be executed every time the client starts. After that it will record the system process PID, then it will detect if the process is still running every 30 seconds. If the process terminates abnormally, "watchdog” will send a signal to system and restart the system. With the help of "watchdog”, the system can run stably for a long time. 
Meanwhile, in order to monitor the network, the system also sets the "heartbeat" mechanism. Client will send a simple POST request to the server every 3 minutes to tell that it is still alive. Once server get the request, it'll record it. If server does not receive a request for a long time, it will send an alarm message to the administrators by WeChat message, email or SMS, notifying them to check what happened.

\section{Gateway Login Automatically}

In some special network environments (such as schools), client need to firstly login the gateway by username and password. Only then client can connect to network. In this system, we realize a separate gateway login module. By pre-set gateway IP, username and password, program will auto post Http request to the gateway server, and with the "heartbeat" mechanism introduced above, we can timely check the gateway login status.

\section{Auto Power Manager}

For this printing system, the realization of automatic power switch function, could not only save energy consumption, but also extend the life of the client machine. By using the "Task Scheduler" in Windows7 [4], we can make the scripts or programs run regularly. In general, the system specifies to sleep at $12 \mathrm{pm}$ and restart in $6 \mathrm{am}$.

\section{E. Local File Automatically Cleaner}

After long-time running, the client will store large numbers of photo files, order files and system logs, which will waste a lot of storage and is not conducive to the protection of user privacy. The system realizes an automatically local file cleaner, aiming at cleaning the big files timely and intelligent. This function will make the system more stable.

\section{RESUlTS AND CONCLUSION}

\section{A. Results}

Currently, the entire system has been promoted in several locations such as campus and office buildings in Beijing, China. The whole system is stable, mature, and has almost no bugs. A screenshot of a running client is shown in Fig. 3.

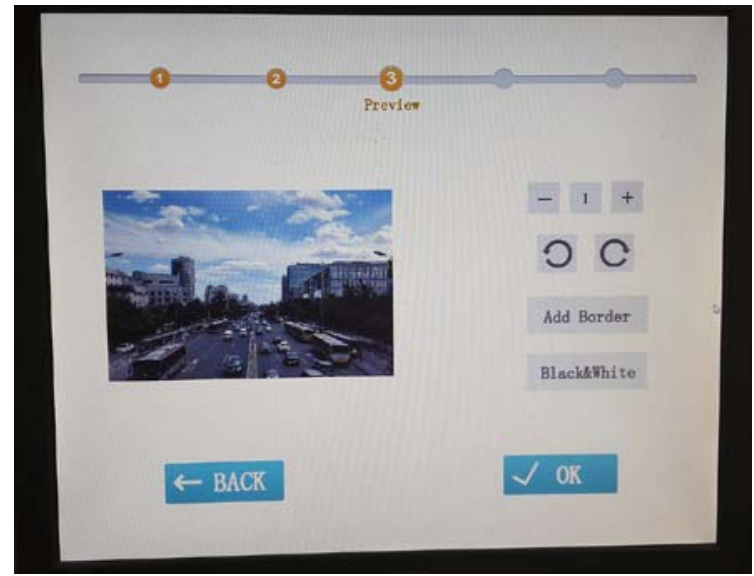

Fig. 3. The process of system

\section{B. Conculsion}

In view of the actual photo printing requirements, this project developed a kind of real-time photo printing system. It is easy use and fast to use, to manage, and has a stable performance. Compared with other printing system in the market, this system has some unique advantages such as supporting for Beijing traffic IC card payment, perfect server management, sensitive early-warning, and various types of photos.

With the help of perfect background management mechanism, our system can carry on large-scale promotion easily. And it's fast and cheap to make system replication and extension.

At present, photo printing has a broad market space and now can hardly meet the needs of users. In the near future, photo printing system will use its unique advantages to challenge the traditional photo and film industry.

\section{REFERENCES}

[1] You-Hong, Li, et al. "Report and investigation model of WeChat popularization in China's colleges and universities in 2015." (2016).

[2] Hector, Basile, and Jacques Hinderer. "pyGrav, a Python-based program for handling and processing relative gravity data." Computers \& Geosciences(2016).

[3] Li, Pengfei, and Kenneth M. Merz. "MCPB. py: A Python Based Metal Center Parameter Builder." Journal of chemical information and modeling(2016).K. Elissa, "Title of paper if known," unpublished.

[4] Kumar, Senthil, et al. "Background Tasks." Windows 10 Development Recipes. Apress, 2016. 213-220. 